treatment, fever had disappeared and two months later, hepatosplenomegaly and pancytopenia had also resolved. The patient has been followed-up without any complication for two years.

1 Berman JD. Human leishmaniasis: clinical, diagnostic, and chemotherapeutic developments in the last 10 years. Clin Infect Dis 1997;24:684-703.

2 World Health Organization. The leishmaniases. Report of WHO expert committee. WHO Tech Rep Ser 1984; no 701

3 Bouree P, Belec L. Leishmaniasis: report of 33 cases and review of the literature. Comp Immun Microbiol Infect Dis $1993 ; 16: 251-65$.

\section{Final diagnosis}

Visceral leishmaniasis (kala azar).

Keywords: leishmaniasis; infection

4 Chance ML. The six diseases of WHO. Leishmaniasis. BMF 1981;283:1245-7.

5 Jeannel D, Tuppin P, Brucker G, Danis M, Gentilini M Imported and autochthonous kala-azar in France. $B M F$ 1991;303:336-8.

6 Badaro R, Falcoff E, Badaro FS, et al. Treatment of visceral leishmaniasis with pentavalent antimony and interferon gamma. N Engl f Med 1990;322:16-21.

\title{
An adolescent girl with abnormal liver profile
}

\author{
Satheesh Nair, C S Pitchumoni
}

A 17-year-old previously healthy high school student who lived in a dormitory was referred to our office by her private physician for evaluation of abnormal liver function tests. She was sexually active with one partner but denied any current or past substance abuse. The patient was not taking any medications or nutritional supplements. Family history was unremarkable. Physical examination revealed scleral icterus and minimal hepatomegaly. Spleen was not palpable. The liver function tests are shown in table 1 . Total leucocyte count was $6.3 \times 10^{9} / 1$ with $53 \%$ lymphocytes. The platelet count was normal. Anti-Hbc IgM antibody was negative, so were anti-HAV IgM and anti-HCV antibodies. HBsAg was negative and anti-HBs antibody was positive. Erythrocyte sedimentation rate was $16 \mathrm{~mm}$ in the first hour. An abdominal sonogram was done to evaluate a persistent elevation in alkaline phosphatase and it showed only hepatomegaly.

Table 1 Liver function tests

\section{Division of Medicine and Gastroenterology, Our Lady of Mercy Medical Center, New York Medical College, 600 East 233 Street, Bronx, NY 10466, USA S Nair C S Pitchumoni}

Correspondence to CS Pitchumoni

\begin{tabular}{|c|c|c|c|c|c|c|}
\hline \multirow[b]{2}{*}{ Duration of illness } & \multirow[b]{2}{*}{ Normal range } & \multirow[b]{2}{*}{ First visit } & \multicolumn{4}{|c|}{ Week } \\
\hline & & & 1 & 3 & 4 & 5 \\
\hline Total bilirubin (mg\%) & $0.2-1.2$ & 1.4 & 2.4 & 1.3 & 1.3 & 1.1 \\
\hline Alanine transaminase $(\mathrm{IU} / \mathrm{ml})$ & $7-49$ & 474 & 322 & 92 & 35 & 13 \\
\hline Aspartate transaminase (IU/ml) & $10-38$ & 470 & 179 & 59 & 31 & 19 \\
\hline Alkaline phosphatase (IU/ml) & $7-128$ & 253 & 494 & 310 & 179 & 117 \\
\hline
\end{tabular}

\section{Questions}

1 What other historical information is important in evaluating this patient?

2 What is the next step in establishing the diagnosis?

3 What is the management of this patient? 


\section{Answers}

QUESTION 1

Any history of infectious mononucleosis (IMN). The clues indicating a probable Epstein-Barr virus (EBV) hepatitis are lymphocytosis (with atypical cells), self-limited transaminitis and a relatively high alkaline phosphatase (normal upper limit for a 17-yearold girl is $128 \mathrm{IU} / \mathrm{l})$. A transient rise in transaminases is seen in $90 \%$ of patients in acute EBV infections. ${ }^{1}$ Transaminase elevation is typically mild (4-5 times the normal) and values more than 10 times the normal should prompt evaluation for other causes. ${ }^{1}$ Interestingly, a persistent and disproportionate elevation of alkaline phosphatase, probably due to cholestasis from an intense periportal mononuclear infiltrate and granuloma formation is sometimes the only clue to EBV hepatitis. A similar picture is seen in cytomegalovirus hepatitis.

Transaminitis does not pose any diagnostic challenges when it occurs as a part of full spectrum of IMN. However, when transaminitis is noted after resolution of typical IMN symptoms or when it occurs in the absence of other features of IMN (as in our patient), diagnosis is often missed, leading to unnecessary investigations including a liver biopsy. Our patient denied any sore throat, but mentioned a recent epidemic of IMN in her dormitory prompting us to look for serological evidence of EBV infection. Atypical lymphocytes seen in her peripheral smear also suggested IMN. Other differential diagnoses which may be considered in a young patient presenting with transaminitis include cytomegalovirus infection, $Q$ fever and leptospirosis, autoimmune hepatitis and drug-induced liver injury. If clinical suspicion is high, a negative anti-HCV antibody test does not rule out an acute hepatitis $\mathrm{C}$, though $\mathrm{HCV}$ RNA may be helpful.

QUESTION 2

EBV serology. A properly timed EBV antigen and antibody estimation is nearly $100 \%$ accurate in making the diagnosis of acute EBV

Table 2 Diagnosis of EBV infection

\begin{tabular}{llllll}
\hline & $V C A I g M$ & $V C A I g G$ & $E A$ & $E B N A \operatorname{Ig} G$ & EBNA IgM \\
\hline Acute infection & ++ & + & + & -- & + \\
Past infection & ++ & -- & $-/+$ & + & $-1+$ \\
Re-activation & + & - & + & + & $-1+$ \\
\hline
\end{tabular}

VCA=viral capsid antigen, EBNA=Epstein Barr nuclear antigen, $\mathrm{Ig}=$ immunoglobulin, EA=early antigen. infection (table 2). The diagnostic serology for acute primary infection is the presence of $\operatorname{IgM}$ antibodies to capsid antigen (IgM VCA). ${ }^{2}$ The diagnosis is further confirmed by demonstrating a falling titre of IgM VCA and seroconversion to antibodies to EBV nuclear antigens. ${ }^{3}$ However, a definitive diagnosis of EBV hepatitis is made by liver biopsy with demonstration of EBV genome in hepatocytes using in situ hybridisation and this is particularly true in cases of post-transplant allograft infection. Liver biopsy is not required in the setting of acute self-limiting hepatitis with a serology conclusive of acute infection.

The serology in our patient was as follows: IgM VCA 647 ELISA units (normal <100) which decreased to 275 after four weeks, a IgM antibody to nuclear antigen 264 ELISA units (normal < 56), early antigen 192 ELISA Units (normal < 110) and a negative IgG antibody to nuclear antigen. These serologic responses are diagnostic of acute EBV infection. Serology for cytomegalovirus was negative. A positive anti$\mathrm{HBs}$ antibody with a negative IgG $\mathrm{HBc}$ antibody suggested prior vaccination. A monospot test for heterophile antibody is a simple initial serological test and is helpful in clinching the diagnosis in patients with typical features of IMN. Monospot test was not performed in our patient since we opted for more specific serology tests in view of the atypical presentation.

\section{QUESTION 3}

No specific treatment is required. EBV hepatitis is a self-limited illness in an immunocompetent host. Though acyclovir and interferon have demonstrated significant antiviral effects in vitro, there is no evidence to support their use in uncomplicated acute illness. ${ }^{4}$ Similarly glucocorticoids are not indicated for acute hepatitis. EBV does not lead to chronic hepatitis in an immunocompetent patient and there is no need for long-term follow-up. However, in a rare patient with defective response of $\mathrm{T}$ cells to asialoglycoprotein receptor, or receptorEBV complex , EBV can trigger an autoimmune type of chronic hepatitis.

Our patient had complete normalisation of liver functions and is doing well after six months.

\section{Final diagnosis}

EBV hepatitis.

Keywords: infectious mononucleosis; Epstein-Barr virus; hepatitis

4 Tynell E, Aurelius E, Brandel A, et al. Acyclovir and prednisone treatment of acute infectious mononucleosis: multicenter double blind placebo controlled study. $\mathcal{F}$ Infect Dis 1996;174:324-31.

5 Vento S, Guella L, Mirandola F, et al. Epstein Barr virus as a trigger for autoimmune hepatitis in susceptible individuals. Lancet 1995;346:608-9. tious mononucleosis. Mil Med 1964;129:533-8. Markin RS. Manifestation of Epstein Barr virus associated disorders in liver. Liver 1994;14:1-13.

infectious mononucleosis. In: virus infections, including E,Wilson JD,Martin JB,Fauci AS, Kasper DL, eds. Harrison's Principles of in 